\title{
MÉTODO AUTOMATIZADO DE IDENTIFICACIÓN Y CARACTERIZACIÓN DE UNIDADES DE PAISAJE
}

\author{
J. ALCÁNTARA-MANZANARES ${ }^{1 *}$, J.M. MUÑOZ-ÁLVAREZ²
}

\begin{abstract}
${ }^{1}$ Departamento de Didáctica de las Ciencias Sociales y Experimentales, Universidad de Córdoba, Facultad de Ciencias de la Educación, Avda. San Alberto Magno s/n, 14071 Córdoba, España. ${ }^{2}$ Departamento de Botánica, Ecología y Fisiología Vegetal, Universidad de Córdoba, Campus Rabanales, Ed. Celestino Mutis, Ctra. Nacional IV Km, 396, 14071 Córdoba, España.
\end{abstract}

RESUMEN. En el marco de un estudio de clasificación del paisaje de la provincia de Huelva (Andalucía) mediante un método objetivo, reproducible y escalable de identificación y caracterización de unidades de paisaje, se ha planteado la utilidad del método para una clasificación a nivel de detalle de uno de los tipos de paisajes resultantes. El tipo de paisaje seleccionado ha sido el denominado Campiñas, con una extensión de $1086 \mathrm{~km}^{2}$. La metodología utilizada ha combinado herramientas propias de los sistemas de información geográfica $(S I G)$ y el análisis multivariante de clasificación TWINSPAN (análisis de especies indicadoras de doble vía), así como el análisis de ordenación DCA (análisis de correspondencias). Las variables utilizadas han sido: usos y coberturas vegetales del suelo, municipios y pendiente. Los resultados obtenidos han sido sometidos a validación mediante análisis discriminante y a un proceso de depuración a nivel gráfico.

Como resultado del estudio se han identificado tres unidades de paisaje dentro del tipo Campiñas, se han delimitado cartográficamente y se han identificado las variables diagnósticas propias de cada una de ellas. La mayoría de las variables indicadoras han sido relativas a los usos del suelo, mientras que la variable de pendiente no ha sido de valor significativo para tal diferenciación. El análisis gráfico de ordenación ha permitido constatar características de las unidades de paisaje no deducibles del TWINSPAN y que complementan la información derivada del mismo. Los resultados del análisis discriminante validan la clasificación realizada. Se justifican las variables utilizadas y se compara la metodología seguida con otros enfoques automatizados, así como los resultados de la clasificación con los obtenidos en la clasificación del Atlas de los Paisajes de España. Por último se concluye la utilidad de la metodología seguida, TWINSPAN, para una clasificación de paisajes a nivel de detalle, analizándose las razones de esta idoneidad.

\section{Automated method for identification and characterization of landscape units}

ABSTRACT. Within the framework of a study aimed at classifying the landscape of Huelva province (Andalusia, Spain) using an objective, reproducible and scalable 
method of identifying and characterising landscape units, we examined the value of this method for the detailed classification of one such landscape unit-designated Campiñas - with a surface area of $1086 \mathrm{~km}^{2}$. The method in question combined tools used in geographical information systems (GIS), multivariate analysis using the TWINSPAN classification (two-way indicator species analysis) and DCA ordination analysis (detrended correspondence analysis). The variables used were land use and plant cover, municipalities, and slope. The results obtained were validated by discriminant analysis and subjected to plot-level debugging.

Three different landscape units were identified within the Campiñas type; these were delimited cartographically, and the distinctive diagnostic variables of each unit were identified. Most indicator variables were linked to land use, while the slope variable was of no significant value for differentiation purposes. Graphic ordination analysis revealed landscape-unit characteristics that could not be deduced from TWINSPAN analysis, and were thus supplementary to the information provided by that analysis. The results of discriminant analysis confirmed validation of the classification. The variables used were justified, and the method employed was compared to other automated approaches; classification results were also compared to those obtained from the classification used in the Atlas of the Landscapes of Spain. It was finally concluded that the TWINSPAN method was valuable for detailed landscape classification, for the reasons examined.

Palabras clave: clasificación del paisaje, análisis multivariante, Twinspan, sistemas de información geográfica (SIG), Huelva (Andalucía).

Key words: landscape classification, multivariate analysis, Twinspan, geographic information systems (GIS), Huelva (Andalucía).

*Correspondencia: Departamento de Didáctica de las Ciencias Sociales y Experimentales, Universidad de Córdoba, Facultad de Ciencias de la Educación, Avda. San Alberto Magno s/n, 14071 Córdoba. España. E-mail: b62almaj@uco.es

\section{Introducción}

La realidad paisajística actual, sobre todo en paisajes tan intensamente manejados como los mediterráneos, requiere un sistema de planificación regional del paisaje, que permita compatibilizar las necesidades socio-económicas con las condiciones geo-biológicas. La planificación necesita a su vez de un sistema de clasificación del paisaje que sea consistente y refleje los patrones naturales, la capacidad potencial y los límites de las unidades naturales, así como la historia de los usos humanos (Haase, 1989). La clasificación del paisaje, por tanto, se presenta como una herramienta fundamental para la 
consecución de los objetivos de protección, gestión y ordenación del paisaje, acordados en el Convenio Europeo del Paisaje (Council of Europe, 2000).

La clasificación del paisaje puede y debe abordarse a varias escalas, pudiendo tomar como marco de referencia, sin ser vinculantes, los límites administrativos (supranacional, nacional, regional y local). Lo ideal sería que los estudios de paisaje realizados a diferentes escalas pudieran integrarse en un sistema jerárquico, de manera que se distinguiera una jerarquía de tipos y unidades de paisaje y pudiera establecerse una relación entre paisajes de distintos niveles, tal y como indica Swanwick (2002).

Un paisaje es considerado una unidad si es posible trazar sus límites y asignarle una función distinta dentro de una matriz o un mosaico de paisajes distintos. Para ello es necesario trabajar a una escala espacial lo suficientemente grande que permita distinguir las unidades de su entorno (Farina, 2006).

El concepto de unidades del paisaje fue desarrollado por primera vez en los años 1950 y 1960. Dichas unidades recibieron el nombre de geocoros, y pueden considerarse como asociaciones o mosaicos de elementos básicos del paisaje. Las propiedades de los geocoros son el resultado de la combinación de los elementos del paisaje que los integran, así como de su disposición en el espacio. Los geocoros -en tanto que sistemas- muestran nuevas propiedades no explicables por la mera suma de las partes (Bastian, 2000). Conceptualmente los geocoros se corresponden con las unidades de territorio -"land unit"- de Zonneveld (1989); entendiendo territorio como sinónimo de paisaje en su significado de "carácter total de una parte de la superficie terrestre" (Von Humboldt). Se define la unidad de territorio como una extensión de territorio que es ecológicamente homogénea a una determinada escala. Para Zonneveld (1989) el enfoque holístico conlleva que el sistema -en este caso el paisaje-constituye una totalidad compleja que puede ser analizada y caracterizada mediante un número relativamente pequeño de características de diagnóstico bien escogidas. Puede completarse esta equivalencia entre distintos términos paisajísticos, introduciendo las expresiones áreas de carácter paisajístico (Landscape Character Areas) y tipos de carácter paisajístico (Landscape Character Types), terminología desarrollada inicialmente en diversos estudios del paisaje llevados a cabo en el Reino Unido (Swanwick, 2002) y posteriormente asumida en el marco del proyecto ELCAI (European Landscape Character Assessment Iniciative), fruto del cual ha sido el mapa de los paisajes de Europa (LANMAP) (Wascher, 2005). Las áreas de carácter paisajístico se definen como "áreas individuales, únicas, áreas geográficas discretas de un particular tipo de paisaje” (Swanwick, 2002); los tipos de carácter paisajístico como: "tipos distintos de paisaje que son relativamente homogéneos en su carácter paisajístico. Son de naturaleza genérica por lo que pueden presentarse en partes diferentes del país, pero dondequiera que aparezcan comparten combinaciones similares de geología, topografía, patrones de drenaje, vegetación y usos del suelo históricos y patrones de asentamiento" (Swanwick, 2002). Una de las diferencias entre áreas y tipos es, pues, que de cada área de carácter paisajístico existe una sola unidad (o, en términos gráficos, un solo polígono) -de ahí que en el nombre de las mismas suelan aparecer términos geográficos que ayudan a su ubicación espacial-, mientras que los tipos, por el contrario, engloban más de una unidad y se distribuyen por distintos ámbitos espaciales. Las áreas suelen ocupar el nivel inferior (a escala de mayor detalle) de las clasificaciones jerárquicas, mientras que los tipos ocupan los niveles superiores. Esta diferen- 
ciación la encontramos en distintas clasificaciones que utilizan una terminología diferente, como, por ejemplo, en el Atlas de los Paisajes de España (Sanz Herráiz et al., 2003). Siempre que el territorio sea de una extensión suprarregional se suelen diferenciar ciertas unidades de paisaje que se corresponden conceptualmente con las áreas y otras que se corresponden con los tipos de carácter paisajístico.

Es necesario diferenciar entre las unidades de paisaje y sus elementos constitutivos, mencionados al principio del párrafo previo. Los elementos básicos del paisaje se han identificado también con otros términos, como ecotopos o biotopos (Troll, 1966; Zonneveld, 1979; Agger y Brandt, 1984). Forman y Godron (1986) los identifican como "landscape elements", elementos del paisaje (incluyendo tanto los de origen antrópico como los de origen natural); consideran que desde una perspectiva ecológica constituyen cada uno de ellos un ecosistema.

Puede pues afirmarse que inherente al concepto actualmente prevalente de paisaje -en tanto que objeto de estudio científico-, se encuentra la posibilidad de realizar una tipología de los paisajes, es decir una clasificación. O, lo que es lo mismo, es posible diferenciar "tipos" de paisajes; esta diferenciación puede realizarse a distintos niveles jerárquicos; y no todos los atributos posibles a utilizar en esta clasificación van a tener el mismo peso (Forman y Godron, 1986), lo que lleva al reconocimiento de las características diagnósticas.

Entre las distintas metodologías que pueden seguirse para la clasificación del paisaje Bunce et al. (1996) diferencian entre: a) enfoques intuitivos, en los que la experiencia individual y las decisiones intuitivas constituyen pilares fundamentales del método, y que constituyen la base de la cartografía tradicional (y, por tanto, la base de una mayoría de los mapas existentes a nivel nacional en Europa (Wascher, 2005)); b) métodos subjetivos formalizados, que constituyen una variante del tipo anterior y c) métodos matemáticos, objetivos, de desarrollo más reciente y que recurren a métodos multivariantes.

En el marco de un estudio de clasificación del paisaje de la provincia de Huelva considerada en su integridad, utilizando métodos multivariantes, se abordó, en una fase ulterior, la clasificación de unidades de paisaje a un nivel jerárquico inferior, utilizando la misma metodología, a fin de analizar la validez del método para esta escala de detalle, lo que constituye el objetivo del presente estudio. A tal fin se seleccionó el tipo de paisaje Campiñas identificado en la clasificación a nivel provincial junto a otros siete tipos distintos (Alcántara, 2008).

\section{Materiales y Métodos}

\section{1. Área de estudio}

El área de estudio se corresponde con la parte del territorio de la provincia de Huelva identificada como tipo de paisaje Campiñas en Alcántara (2008) (Fig. 1). El territorio ocupa una superficie de $1086 \mathrm{~km}^{2}$, con un relieve suave alomado, altitudes mayoritariamente inferiores a los $200 \mathrm{~m}$, pendientes predominantes entre 7-15\%, dominando en la litología las margas, y mostrando como usos del suelo mayoritarios -en orden descendente- a los cultivos herbáceos en secano, el olivar, el viñedo y los cítricos. 


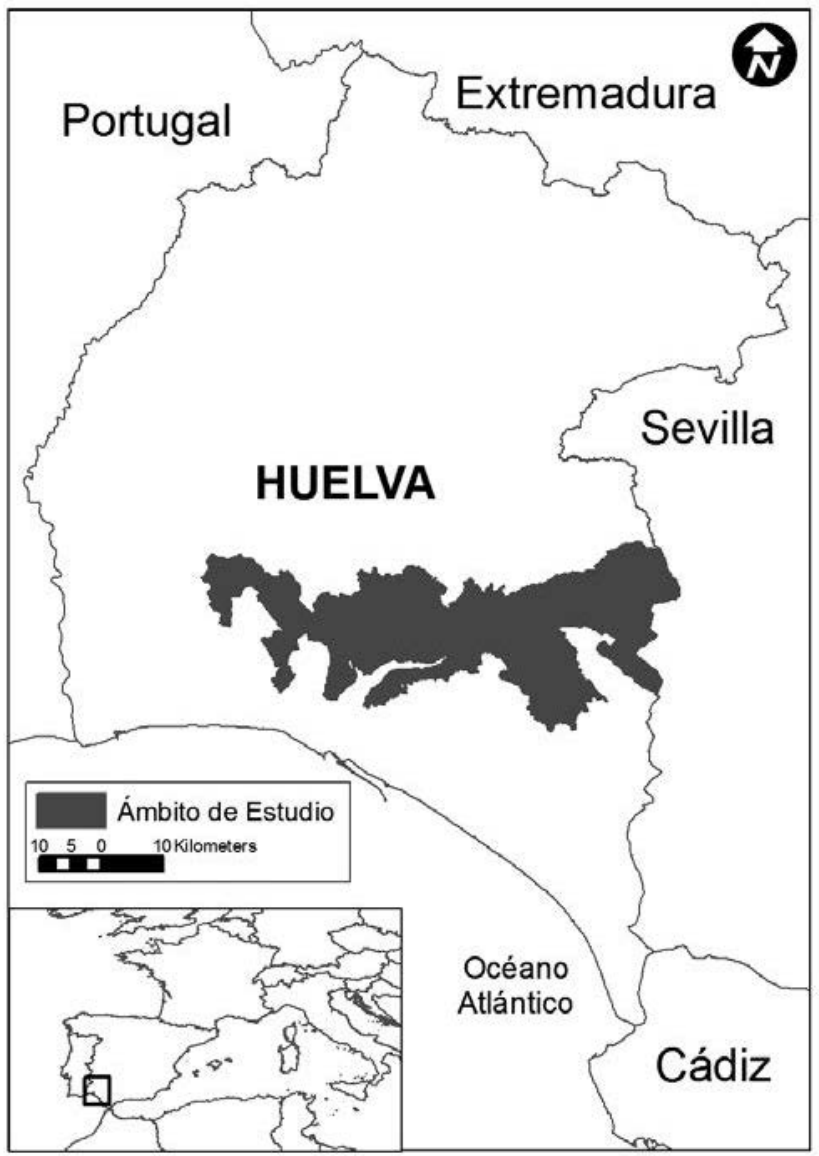

Figura 1. Mapa del área de estudio, Campiñas (en gris), en Huelva (Andalucía, España).

\subsection{Obtención de los datos}

Se ha dividido el área de estudio en cuadrículas georreferenciadas de $1 \mathrm{~km} \mathrm{x} 1 \mathrm{~km}$. Se han obtenido 1301 cuadrículas, a las que se ha asociado información relativa a las variables usos del suelo y coberturas vegetales, pendiente (variable de relieve) y municipios (variable de referencia espacial). Para cada una de estas variables se han diferenciado las siguientes clases:

1) 46 clases de usos del suelo y coberturas vegetales (Fig. 2). Se han obtenido a partir de la Cartografía digital de Usos del Suelo y Coberturas Vegetales de Andalucía, a escala 1:25 000, (provincia de Huelva), existente en la Red de Información Ambiental de Andalucía (REDIAM). Se ha realizado una reunificación en 46 clases.

2) 6 clases de pendiente (Fig. 3). Se han obtenido a partir de un modelo digital de elevaciones. Se ha utilizado la siguiente división en clases: clase $1,<3 \%$, clase 2, 3-7\%; clase 3, 7-15\%; clase 4, 15-30\%; clase 5, 30-45\%; clase 6, >45\%. 

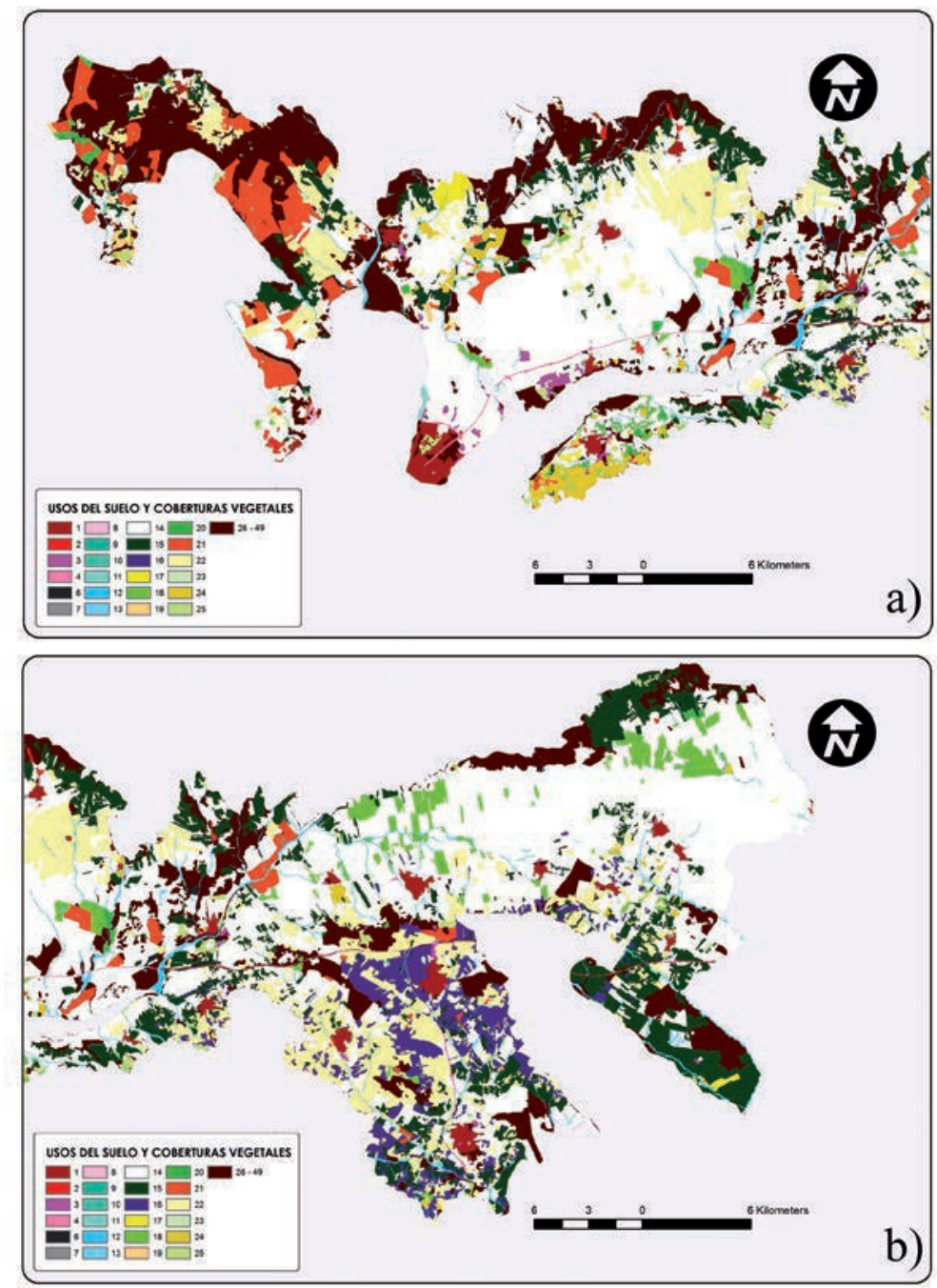

Figura 2. Mapa de usos del suelo y coberturas vegetales del territorio correspondiente al tipo de paisaje Campiñas. a) Mitad occidental; b) mitad oriental. 1. Tejido Urbano; 2. Urbanizaciones; 3. Zonas Industriales, Comerciales y Otras Infraestructuras Técnicas; 4. Autovías, Autopistas, Enlaces Viarios, Comp. Ferroviarios y Aeropuertos; 6. Zonas Mineras; 7. Escombreras, Vertederos y Zonas en Construcción; 8. Zonas Verdes y Espacios de Ocio; 9. Marisma Colonizada por Vegetación; 10. Marisma Reciente sin Vegetación;

11. Salinas y Parques de Cultivo Acuáticos; 12. Ríos y Cauces; 13. Embalses, Balsas y Lagunas Continentales; 14. Cultivos Herbáceos en Secano; 15. Cultivos Leñosos en Secano: Olivar; 16. Cultivos Leñosos en Secano: Viñedo; 17. Otros Cultivos Leñosos en Secano; 18. Cultivos Herbáceos en Regadío; 19. Cultivos Forzados Bajo Plástico; 20. Cultivos Leñosos en Regadío; 21. Cítricos; 22. Áreas Agrícolas Heterogéneas en Secano; 23. Áreas Agrícolas Heterogéneas en Regadio; 24. Mosaicos de Secanos y Regadíos; 25. Mosaicos de Cultivo con Vegetación Natural; 26-49. Usos del suelo y coberturas vegetales pertenecientes al LandCover forestal. 


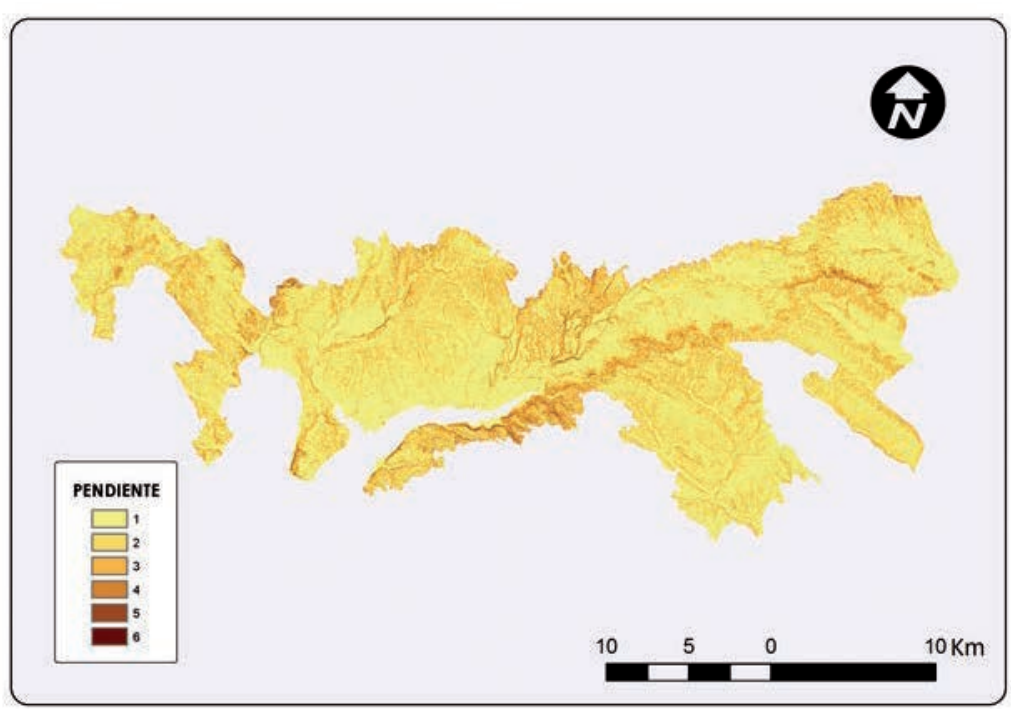

Figura 3. Mapa de clases de pendiente del territorio correspondiente al tipo de paisaje Campiñas.

Clases de pendiente: $1 .<3 \% ; 2.3-7 \% ; 3.7-15 \% ; 4.15-30 \% ; 5.30-45 \% ; 6 .>45 \%$.

3) 24 clases de municipios (Fig. 4). Se han localizado 24 municipios, en su totalidad o parcialmente, dentro del tipo de paisaje Campiñas.

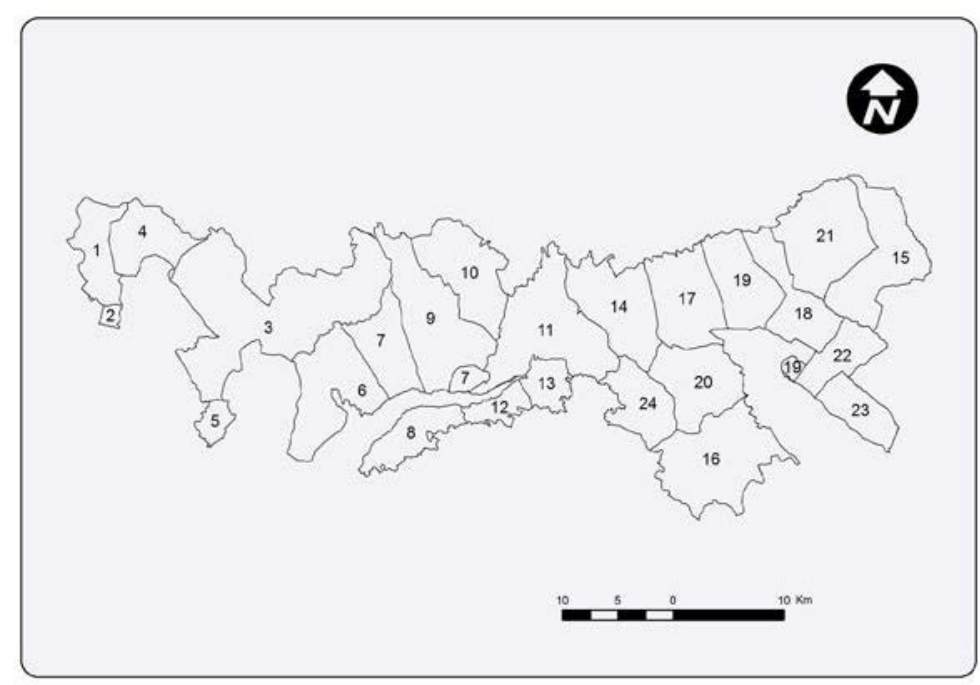

Figura 4. Mapa de municipios del territorio correspondiente al tipo de paisaje Campiñas. Nombres de los municipios: 1. Villanueva de los Castillejos; 2. Cartaya; 3. Gibraleón; 4. San Bartolomé de la Torre; 5. Aljaraque; 6. Huelva; 7. San Juan del Puerto; 8. Moguer; 9. Trigueros; 10. Beas; 11.

Niebla; 12. Lucena del Puerto; 13. Bonares; 14. Villarrasa; 15. Escacena del Campo; 16. Almonte; 17. Palma del Condado; 18. Manzanilla; 19. Villalba del Alcor; 20. Bollullos Par del Condado; 21.

Paterna del Campo; 22. Chucena; 23. Hinojos; 24. Rociana del Condado. 


\subsection{Validación de la clasificación}

Para la validación de la clasificación se han utilizado variables relativas a: litología; altitud (variable de relieve); distancia a la costa (variable de referencia espacial); índice de forma (variable relativa al patrón del paisaje, aporta información sobre la estructura interna del paisaje -paisaje como criptosistema: parte oculta subyacente del paisaje (González Bernáldez, 1981)); y series de vegetación (variable que refleja la vegetación potencial y, en consecuencia, determinadas características climáticas y edáficas del territorio). Para cada una de estas variables se han diferenciado las siguientes clases:

a) 6 clases litológicas (Fig. 5). La información sobre la litología se ha obtenido a partir de la Cartografía Temática de Andalucía, Medio Físico, a escala 1:100 000. Se han diferenciado 6 clases correspondientes a 5 tipos de litología y una a superficies de agua.

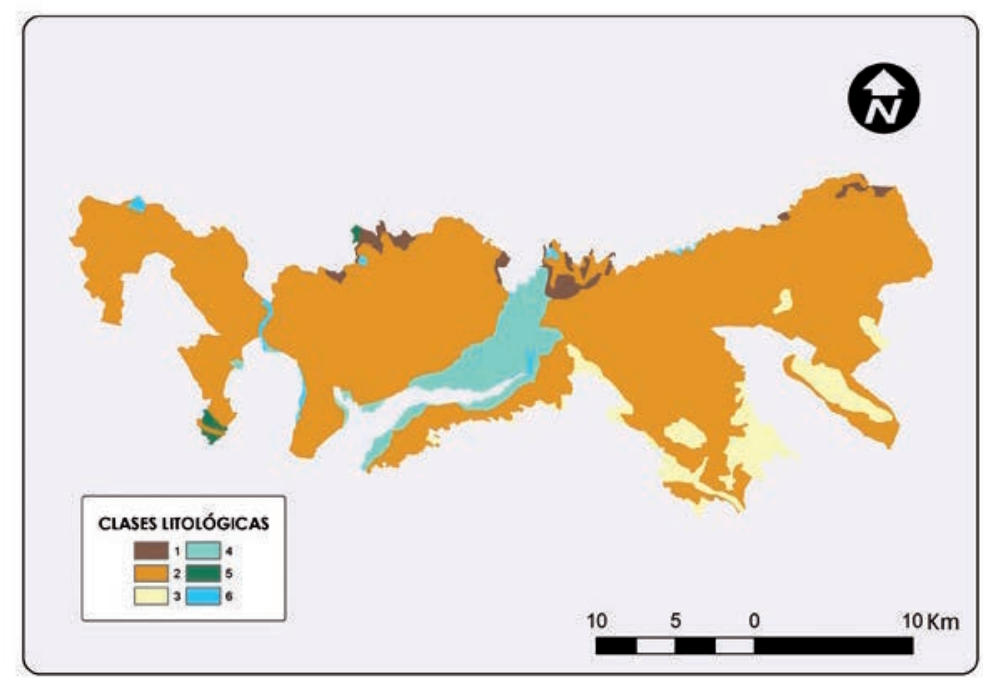

Figura 5. Mapa litológico del territorio correspondiente al tipo de paisaje Campiñas. Clases litológicas: 1. Pizarras y cuarcitas; 2. Margas; 3. Arenas; 4. Limos; 5. Gravas y conglomerados; 6. Lámina de agua.

b) 5 clases de altitud (Fig. 6). Los datos sobre altitud se han obtenido a partir del Modelo Digital de Elevaciones (MDE), de Andalucía, a escala 1:50 000, perteneciente a las cartografías temáticas de la REDIAM. Para el presente estudio se ha considerado conveniente establecer rangos de $50 \mathrm{~m}$, habiéndose obtenido un total de 5 clases.

c) 5 clases de distancia a la costa (Fig. 7). Las clases se han obtenido tomando como referencia la línea de costa de la provincia de Huelva y estableciendo un buffer con un rango de $10 \mathrm{~km}$.

d) 14 clases de índice de forma (SI) (Fig. 8). El índice se ha calculado a partir de los usos del suelo y coberturas vegetales. Para su cálculo se ha dividido, para cada polígono, el perímetro entre la raíz cuadrada del producto del área por $\Pi$ (constante de ajuste) 
(McGarigal y Marks, 1994). Se han establecido 19 rangos, de los cuales solo 14 están representados en el área de estudio.

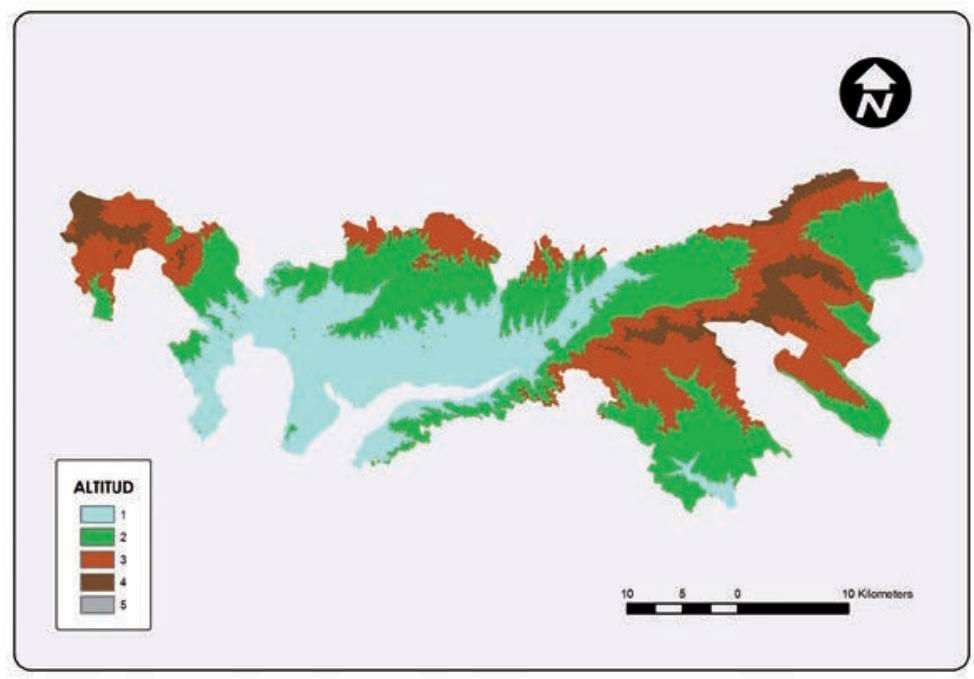

Figura 6. Mapa de 5 clases de altitud del territorio correspondiente al tipo de paisaje Campiñas. Clases de altitud: 1.0 - $50 \mathrm{~m} ; 2.50$ - $100 \mathrm{~m} ; 3.100-150 \mathrm{~m} ; 4.150-200 \mathrm{~m} ; 5 .>200 \mathrm{~m}$.

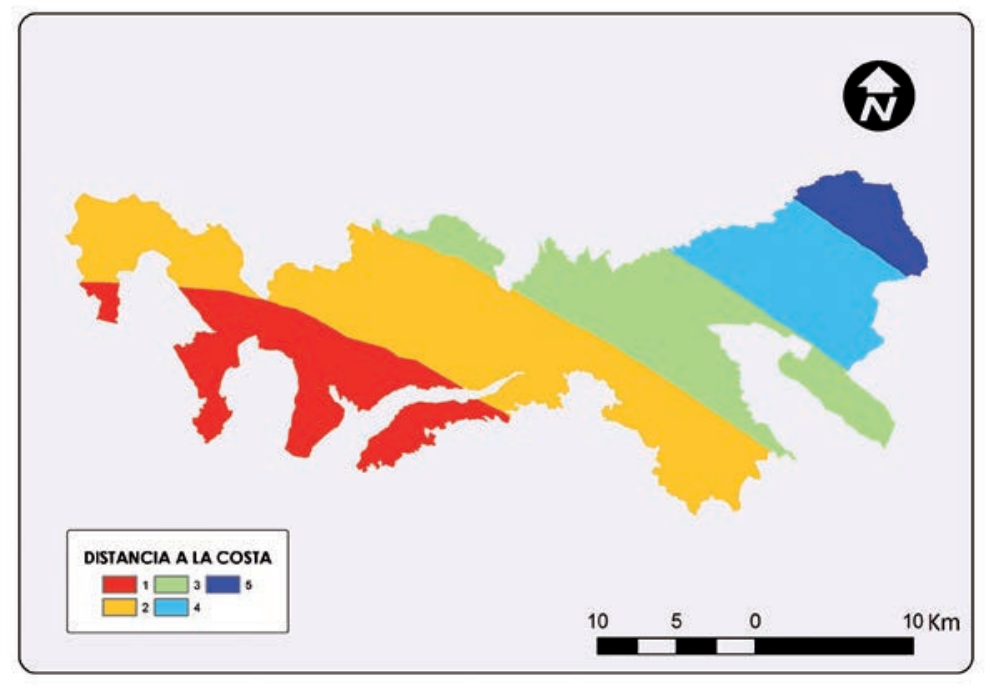

Figura 7. Mapa de clases de distancia a la costa del territorio correspondiente al tipo de paisaje Campiñas. Clases de distancia a la costa: 1.10 - 20 km.; 2.20 - 30 km.; 3.30 - 40 km.; 4. 40 - $50 \mathrm{~km}$.; $5.50-60 \mathrm{~km}$. 


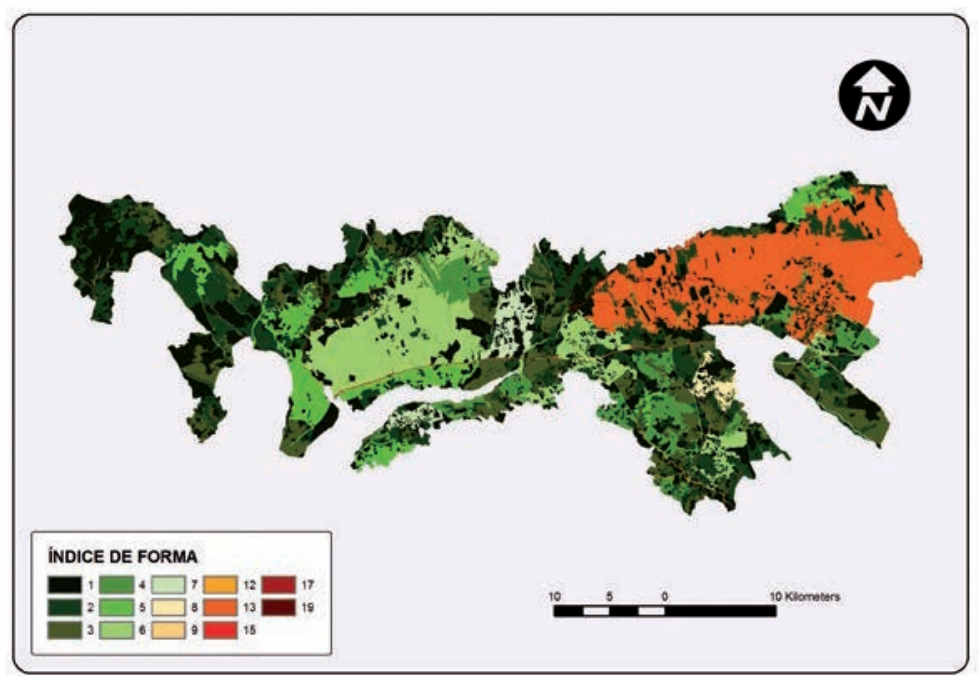

Figura 8. Mapa de clases del Índice de Forma (SI) del territorio correspondiente al tipo de paisaje Campiñas. Clases del Índice de Forma: 1. SI 1-2; 2. SI 2-3; 3. SI 3-4; 4. SI 4-5; 5. SI 5-6; 6. SI 6-7; 7. SI 7-8; 8. SI 8-9; 9. SI 9-10; 10. SI 10-11; 11. SI 11-12; 12. SI 12-13; 13. SI 13-14; 14. SI 14-15; 15. SI 15-16; 16. SI 16-17; 17. SI 17-18; 18. SI 18-19; 19. SI 19-20.

e) 6 clases de series de vegetación (Fig. 9). Las series de vegetación se han delimitado a partir del Mapa de Series de Vegetación de España a escala 1:400 000 (Rivas-Martínez, 1987).

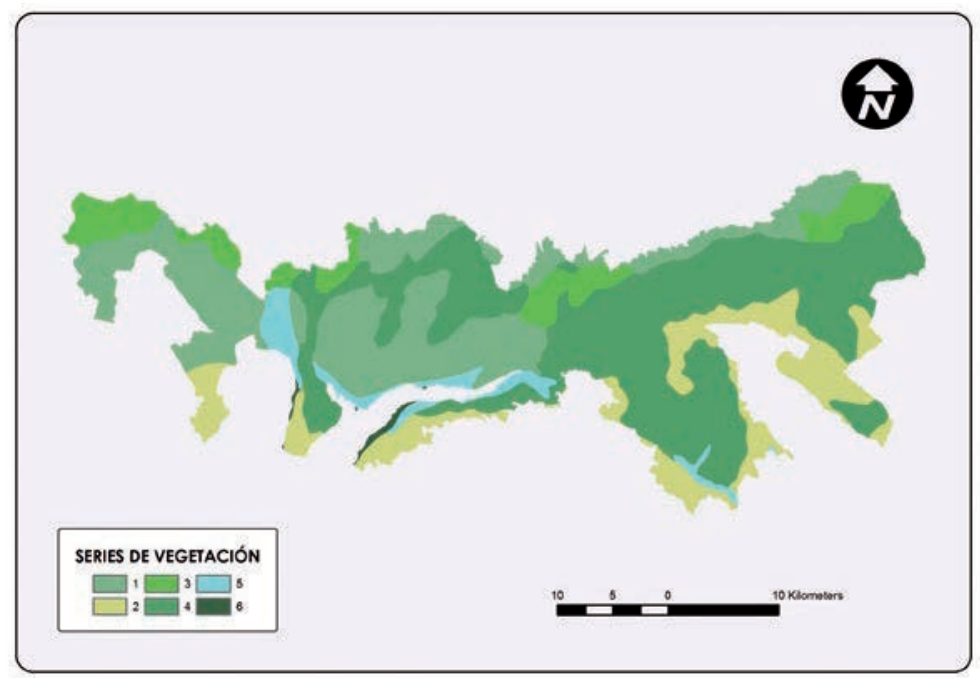

Figura 9. Mapa de series de vegetación del territorio correspondiente al tipo de paisaje Campiñas. Nombres de las series de vegetación: 1. Oleo-Querceto suberis sigmetum, faciación típica mariánico-monchiquense. 2. Oleo-Querceto suberis sigmetum, faciacion mariánico pacense y Gaditanoonubense sobre arenales con Halimium halimifolium. 3. Myrto-Querceto rotundifoliae sigmetum,

faciación típica. 4. Smilaci mauritanicae-Querceto rotundifoliae sigmetum, faciación típica.

5. Geomegaseries riparias mediterráneas y regadios. 6. Geomacroserie de los saladares y salinas. 


\subsection{Identificación de Unidades de Paisaje}

\subsubsection{Análisis Multivariante de Clasificación: TWINSPAN}

Para la clasificación de las cuadrículas (previa estandarización 0-1) se ha utilizado como método multivariante de clasificación el TWINSPAN (Two Way Indicador Species Analysis, Kent y Coker, 1992), mediante el software PCOrd 4.0. El TWINSPAN pertenece al grupo de métodos de clasificación jerárquicos, divisivos y politéticos. Partiendo del conjunto de muestras a clasificar realiza sucesivas divisiones dicotómicas, estableciendo dos grupos en cada una, a partir de los valores de las variables. Estos grupos se disponen a un lado y otro (positivo uno y negativo el otro) del eje correspondiente de ordenación. Previamente cada variable de tipo cuantitativo es dividida en variables de tipo cualitativo - pseudovariables-, utilizando ciertos niveles de corte. Los niveles de corte utilizados en este estudio han sido $0.02,0.05,0.10,0.20,0.50,0.70$ y 0.90 . La presencia diferencial de pseudovariables distingue entre sí a los grupos formados en cada división. Existe un valor que cuantifica esta diferencia: el valor indicador (I). Se asume como regla básica que la pseudovariable con mayor valor indicador cuenta como el valor indicador global para la variable.

Los grupos establecidos por el TWINSPAN son descritos por sus variables indicadoras y sus variables preferenciales. Las variables indicadoras son aquellas cuyo valor indicador esté comprendido entre: $+0.5<\mathrm{I}<+1$ y $-0.5>\mathrm{I}>-1$. Una variable es considerada como preferencial para un grupo u otro de la dicotomía, cuando la probabilidad de que se presente en un grupo es mayor del doble de la probabilidad de que se presente en el otro, siendo su valor indicador: $-0.5<\mathrm{I}<+0.5$. Un valor de +1 para una pseudovariable implica que está presente en todas las cuadrículas de uno de los dos grupos que se diferencian en cada división dicotómica y falta en todas las del otro grupo; mientras que si fuera de -1, estaría presente en todas las del otro grupo, etc. Se trataría pues de la mejor pseudovariable que puede encontrarse para diferenciar un grupo de otro. Para el análisis de las unidades de paisaje generadas en los distintos niveles de división se han utilizado las variables indicadoras y las variables preferenciales de mayor entidad.

\subsubsection{Análisis Multivariante de Ordenación: DCA}

El análisis multivariante de ordenación utilizado ha sido el DCA (Detrended Correspondence Análisis, Kent y Coker, 1992), mediante el software CAP 3.0. Se han interpretado los dos primeros ejes.

El DCA ha permitido analizar la posición que ocupan las cuadrículas en el espacio de ordenación determinado por las variables y dibujar los grupos formados en dicho espacio de ordenación (McGarigal et al., 2000). Ello ha posibilitado corroborar los clusters formados en la clasificación TWINSPAN y establecer grados de diferencia entre clusters, según la distancia existente entre los mismos (Kent y Coker, 1992).

\subsubsection{Post-procesado de la Clasificación: Depurado}

Se ha procedido al depurado de los grupos generados por el TWINSPAN para la identificación final de las unidades de paisaje. Este proceso ha consistido en: 
a) Sustituir la estructura reticular de los límites entre unidades de paisaje, tomando como referencia, mediante superposición, las variables indicadoras resultantes del TWINSPAN.

b) Eliminar todas aquellas cuadrículas aisladas dentro de otra unidad de paisaje (isleos) cuya identificación cumpliera criterios matemáticos pero no paisajísticos.

\subsubsection{Validación de la clasificación TWINSPAN mediante métodos multivariantes: Análisis Discriminante}

El método multivariante elegido para la validación de la clasificación TWINSPAN obtenida ha sido el Análisis Discriminante. Para el Análisis Discriminante se ha utilizado un método no paramétrico, debido a la naturaleza no normal de los datos: $k$ Nearest Neighbour $(\mathrm{k}=10)$. El paquete estadístico utilizado ha sido S.A.S. (1990). Para que la validación sea significativa, las variables utilizadas en el Análisis Discriminante han de ser diferentes a las empleadas en la clasificación TWINSPAN (Legendre y Legendre, 1998). Por tanto, se ha generado una base de datos con las variables indicadas en el apartado anterior (validación de la clasificación), las 1301 cuadrículas como elementos a clasificar, y la clasificación TWINSPAN como clasificación observada.

La bondad de los resultados del Análisis Discriminante ha sido a su vez evaluada mediante validación cruzada, utilizando el estimador Jack Knife (Manly, 1997; Muñoz Serrano, 2003).

\section{Resultados}

Los resultados del TWINSPAN (Fig. 10) permiten el reconocimiento de 3 grupos de cuadrículas, cuya distribución en el ámbito estudiado se muestra en la Fig. 11. Son tres unidades de extensión dispar, abarcando el grupo de cuadrículas 2 un $63.7 \%$ del total de cuadrículas analizadas. La discriminación de estos tres grupos se establece en base a variables de usos del suelo y coberturas vegetales, y municipios; la variable pendiente no es de utilidad para esta diferenciación. La mayor importancia de los usos del suelo y coberturas vegetales se evidencia si se considera que de las diez variables diferenciales (Fig. 10), siete son variables de usos del suelo y tres de municipios. Esta mayor capacidad discriminante se ve ratificada si se atiende al valor de las variables: solamente aparecen dos que puedan identificarse como variables indicadoras, tal y como se han definido en material y métodos; ambas son variables de usos del suelo (C16, cultivos leñosos en secano: viñedos [ $\mathrm{I}=-0.79]$ y C22, áreas agrícolas heterogéneas en secano [I = -0.57].

En el primer nivel dicotómico las variables más discriminantes son: cultivos herbáceos en secano (C14, I = -0.48; presente en el 18.5\% de las cuadrículas del grupo 1 (G1) [265] y en el $66.5 \%$ de las cuadrículas del otro grupo de este primer nivel [1036]) y el municipio de Gibraleón (M3, I = 0.46; presente en el 52.1\% de cuadrículas del G1 [265] y en el 5.6\% de las cuadrículas del otro grupo [1036]). 


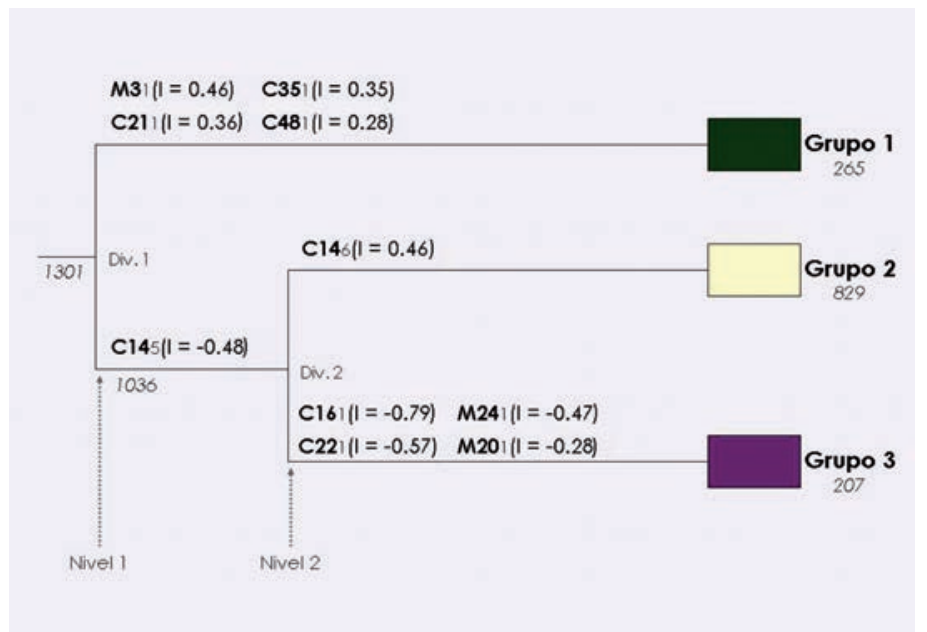

Figura 10. Dendrograma representando la clasificación TWINSPAN de las cuadrículas. Se indican los sucesivos niveles y divisiones del TWINSPAN. Los niveles de abundancia relativa de las variables indicadoras (pseudovariables) se presentan en letra pequeña en escala ordinal $(1,0-2 \% ; 2,2-5 \%$; 3, 5-10\%; 4, 10-20\%; 5, 20-50\%; 6, 50-70\%; 7, 70-90\%; 8, 90-100\%). I=

Valor indicador. En cursiva, número de muestras de cada grupo. En negrita, se recogen las variables indicadoras identificadas como tales en la salida del programa: M3. Gibraleón; C21. Cítricos; C35. Pastizales no arbolados; C48. Formaciones de matorral disperso; C14. Cultivos herbáceos en secano; C16. Cultivos leñosos en secano: Viñedo; C22. Áreas agrícolas heterogéneas en secano; M24. Rociana del Condado; M20. Bollullos Par del Condado.

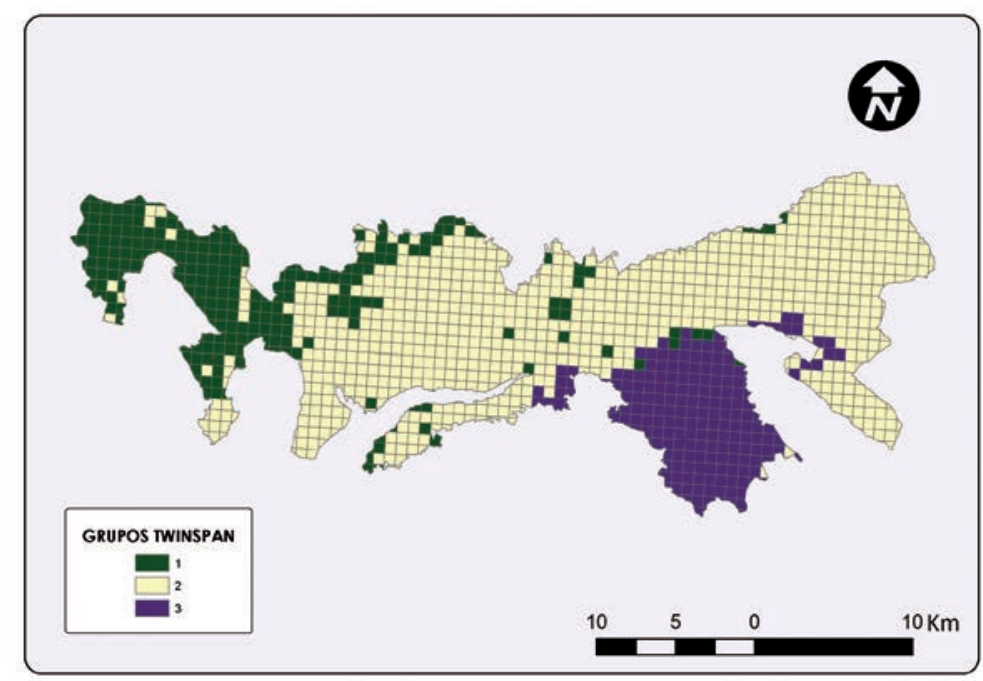

Figura 11. Distribución en el territorio de las cuadrículas adscritas a los grupos derivados del TWINSPAN. 
En el segundo nivel dicotómico los mayores valores corresponden a: cultivos leñosos en secano: viñedo (C16, I = -0.79; en el 85.5\% de cuadrículas del grupo 3 (G3) y en el 5.6\% de las del grupo 2 [G2]); áreas agrícolas heterogéneas en secano (C22, I = -0.57; en el $88.4 \%$ de cuadrículas del G3 y en el $31.12 \%$ de las del G2; y cultivos herbáceos en secano $(\mathrm{C} 14, \mathrm{I}=0.46$; en el $53.7 \%$ de cuadrículas del G2 y en el 7.3\% de las del G3).

Profundizando en el significado de estos valores indicadores, en relación a la variable de los viñedos $(\mathrm{C} 16, \mathrm{I}=-0.79)$, cabe concretar que el valor tan alto de la pseudovariable 1 de C16 (pseudovariable que implica la presencia de viñedos en al menos un $2 \%$ de la superficie de la cuadrícula) deriva de su presencia en 175 de 207 cuadrículas del G3 y tan solo 46 de 829 del G2. Luego el valor indicador elevado de una variable pone de manifiesto su alta frecuencia en un grupo y, en paralelo, una presencia reducida de la misma en el otro grupo. Si se considera además que esta pseudovariable 1 de C16 está presente únicamente en 4 de las cuadrículas del G1, el resultado global (G3: 175/207 cuadrículas y 50/1094 cuadrículas G1 y G2 en conjunto) pone de relieve su valor diagnóstico frente a las unidades de paisaje 1 y 2 , valor diagnóstico que deriva en parte de la fidelidad de esta variable al grupo 3.

Una fidelidad similar se da entre la pseudovariable 1 de cítricos $(\mathrm{C} 21, \mathrm{I}=0.36)$ y el G1. Esta variable se presenta únicamente en 69 cuadrículas del total de 1036 que conforman el otro grupo de este nivel 1 del TWINSPAN (Fig. 10), luego su presencia está casi tan restringida al G1 como estaba la presencia de los viñedos (C16) al G3. La diferencia tan notable en su valor indicador $(\mathrm{I}=0.36$ para $\mathrm{C} 21$ frente a $\mathrm{I}=-0.79$, para C16) se debe a que se presenta únicamente en 113 de las 265 cuadrículas del G1 (42.6\% de las cuadrículas). Puede, pues, apreciarse cómo el valor indicador tiene dos componentes: el grado en que una variable se restringe a un grupo de los dos que se generan en cada nivel de división y el grado de frecuencia con que se presenta en las cuadrículas de tal grupo.

Las dos salidas gráficas del DCA (Figs. 12 y 13) respaldan gráficamente los resultados del dendrograma de la Fig. 9. La Fig. 12 muestra al G3 como al grupo más homogéneo en su distribución en el espacio bidimensional de la gráfica determinado por las variables. La mayor proximidad entre las cuadrículas del mismo no es sino un reflejo de su mayor similitud en relación a los valores de las variables que dan consistencia e independencia al grupo (y que presentan los mayores valores indicadores del análisis, -0.79 y -0.57). Es nítida su diferenciación del G1 en relación al eje 2. Ambos grupos constituyen los extremos de variación respecto a este eje, del total de cuadrículas estudiadas. La distribución de las cuadrículas respecto del eje 1, permite discriminar al G2 tanto del G1 como del G3. En cuanto al G1, a tenor de su distribución en el espacio bidimensional, cabe identificarlo como el más heterogéneo (si se toma en consideración la diferencia entre el número de cuadrículas del mismo, 265, y el número de cuadrículas del G2, 829, que es el que, en sentido estricto, presenta mayor dispersión en la gráfica).

La distribución de las variables indicadoras y preferenciales en el espacio muestral (cuadrículas) (Fig. 13) corrobora y explicita la diferenciación de los tres grupos en relación a los ejes 1 y 2 . Permite además comprender el peso de cada variable en 
la ordenación y clasificación de las muestras. Así, por ejemplo, atendiendo a las cuatro variables diferenciales del G1, se aprecia que su ubicación en el espacio muestral (Fig. 13) las distribuye en dos grupos: M3 y C21 se sitúan más juntas entre sí por debajo del eje de abcisas, mientras que C35 y C48 quedan agrupadas coincidiendo con este eje. El análisis comparado de la distribución de usos del suelo (Fig. 2), municipios (Fig. 4) y grupos Twinspan (Fig. 11), da sentido a esta disposición, al detectarse una cierta coincidencia en la distribución en el territorio entre las mayores extensiones de cítricos (C21) y la parte del municipio de Gibraleón (M3) presente en las cuadrículas del G1. En cuanto a las variables C35 (pastizales no arbolados) y C48 (formaciones de matorral disperso) al no estar representadas independientemente en la Fig. 2, tan solo cabe interpretar su distancia a C21 y M3, constatando su predominio sobre los cítricos en la mitad más occidental de este grupo de cuadrículas, situadas ya fuera del término de Gibraleón. Un análisis similar permite comprender la proximidad en el G3, entre las variables M20 (Bollullos Par del Condado) y C16 (cultivos leñosos en secano: viñedo), así como entre M24 (Rociana del Condado) y C22 (áreas agrícolas heterogéneas en secano).

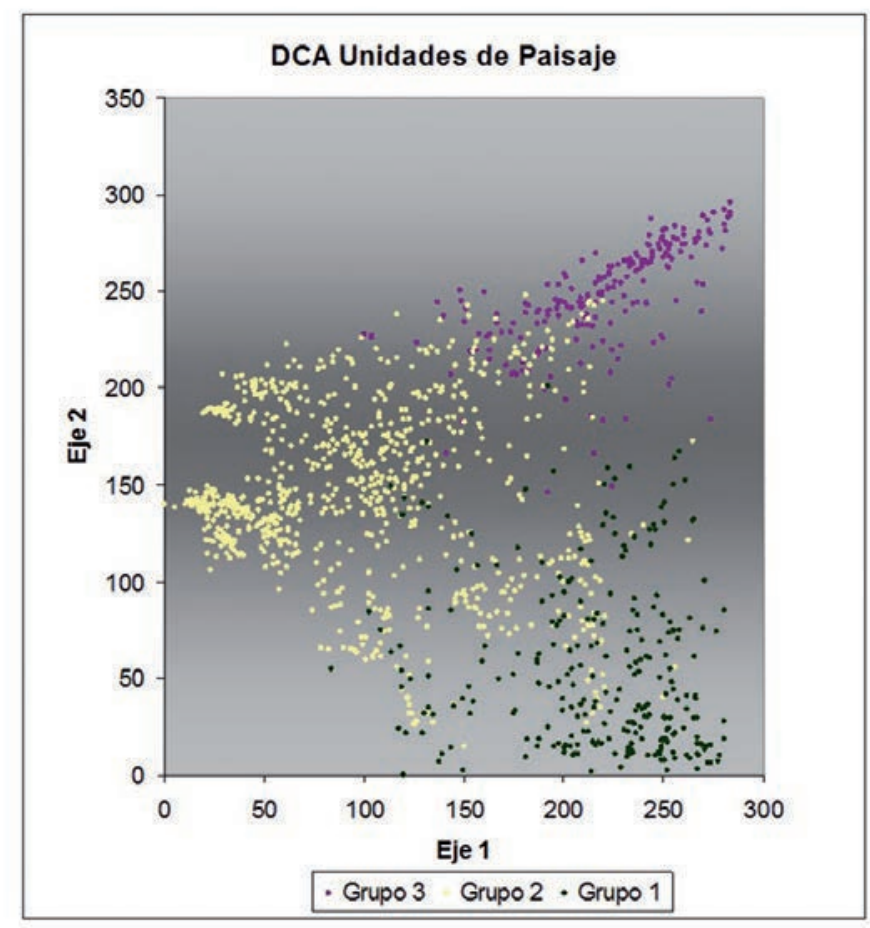

Figura 12. Resultados del DCA: cuadrículas. La correspondencia con los grupos del TWINSPAN se muestra con los colores identificativos para las unidades de paisajes. 


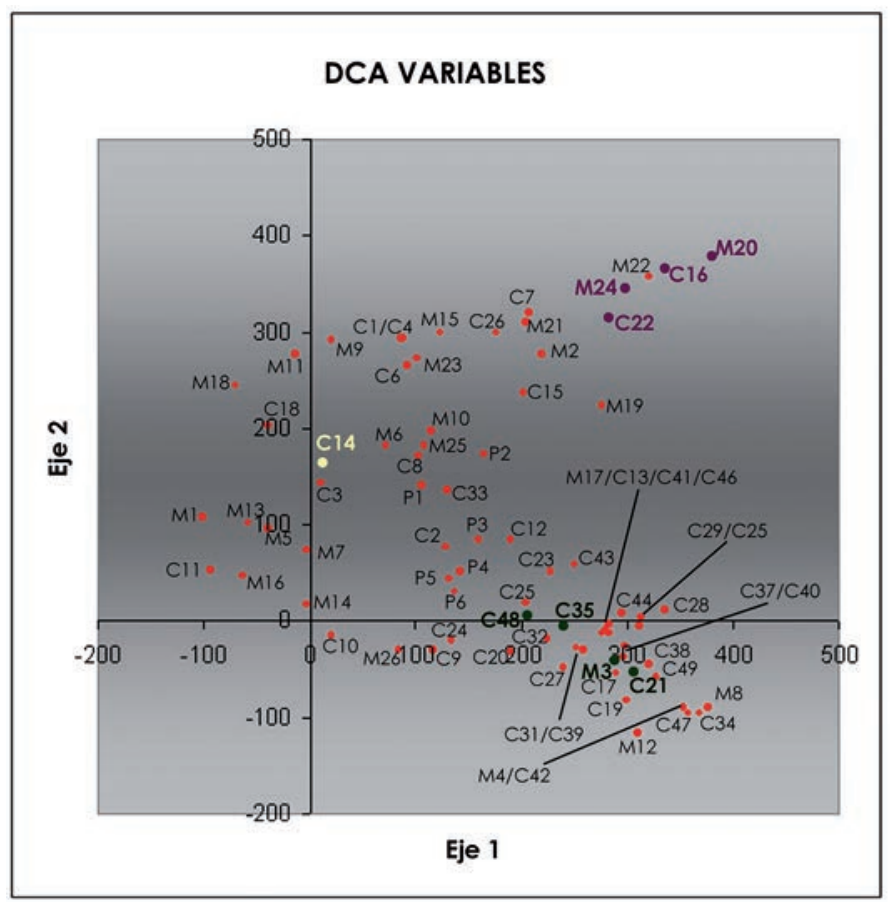

Figura 13. Resultados del DCA: variables. Las variables indicadoras de los grupos derivados del TWINSPAN se identifican con su color correspondiente.

Como resultado del depurado, han quedado delimitadas las unidades de paisaje tal y como se muestra en la Fig. 14. La unidad de paisaje 1 aparece mayoritariamente en la parte más occidental del área de estudio (Foto 1). Se encuentra caracterizada por usos del suelo y coberturas no forestales (C35, pastizales no arbolados, y C48, matorral disperso) (Figs. 2 y 10) y por el cultivo de cítricos (C21). La desembocadura del río Odiel, tanto por la disponibilidad de agua, como por la suavización de las temperaturas, puede explicar la elevada extensión de este tipo de cultivo en este grupo. Las citadas características del G1 imprimen una impronta paisajística tal, que lo hace fácilmente distinguible del resto del territorio. El G2 se extiende por la banda central del territorio, se caracteriza por los cultivos herbáceos en secano (C14) de gran extensión, que otorgan al grupo gran homogeneidad y continuidad (Foto 2). Salvo en zonas periféricas, es el grupo más llano de los tres, dando lugar a amplias cuencas visuales de monocultivo extensivo de cereal. El G3, presenta, en general, un relieve algo menos suave que el G2 y algo más que el G1, caracterizándose por los cultivos leñosos en secano: viñedos (C16) y las áreas agrícolas heterogéneas en secano (C22), y ocupando la mayor parte del Sur del territorio estudiado. El G3 presenta un paisaje mucho más heterogéneo que el G2, siendo los viñedos y los mosaicos de manchas de viñedos, olivares y cultivos herbáceos, junto con vestigios de vegetación natural en los linderos, su imagen más característica (Foto 3). 


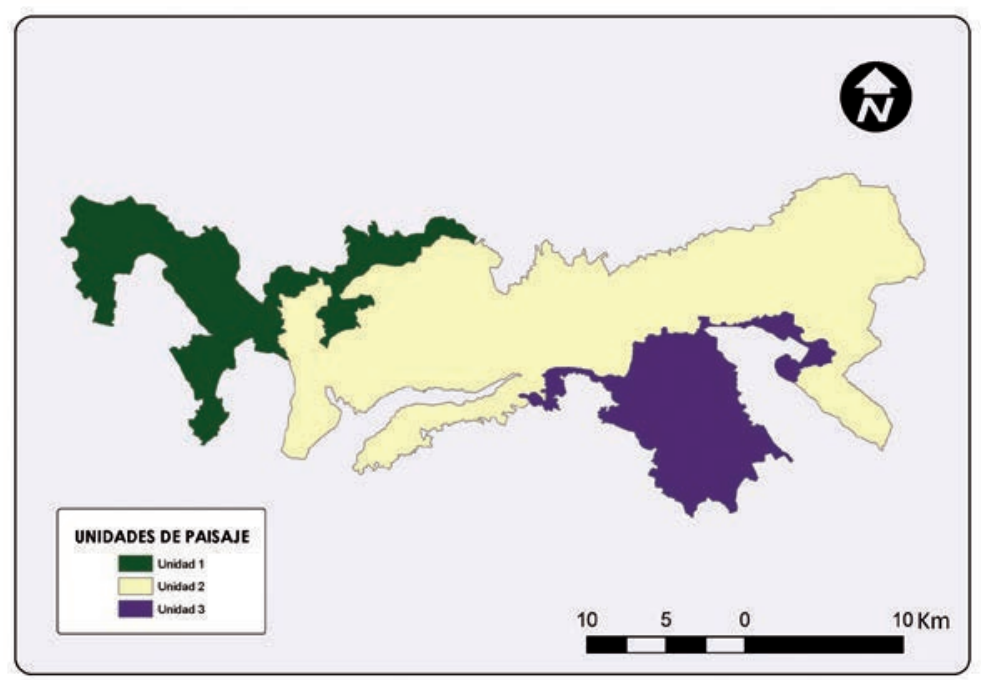

Figura 14. Unidades Paisajísticas o Áreas de Carácter Paisajístico propias del tipo de paisaje Campiñas.

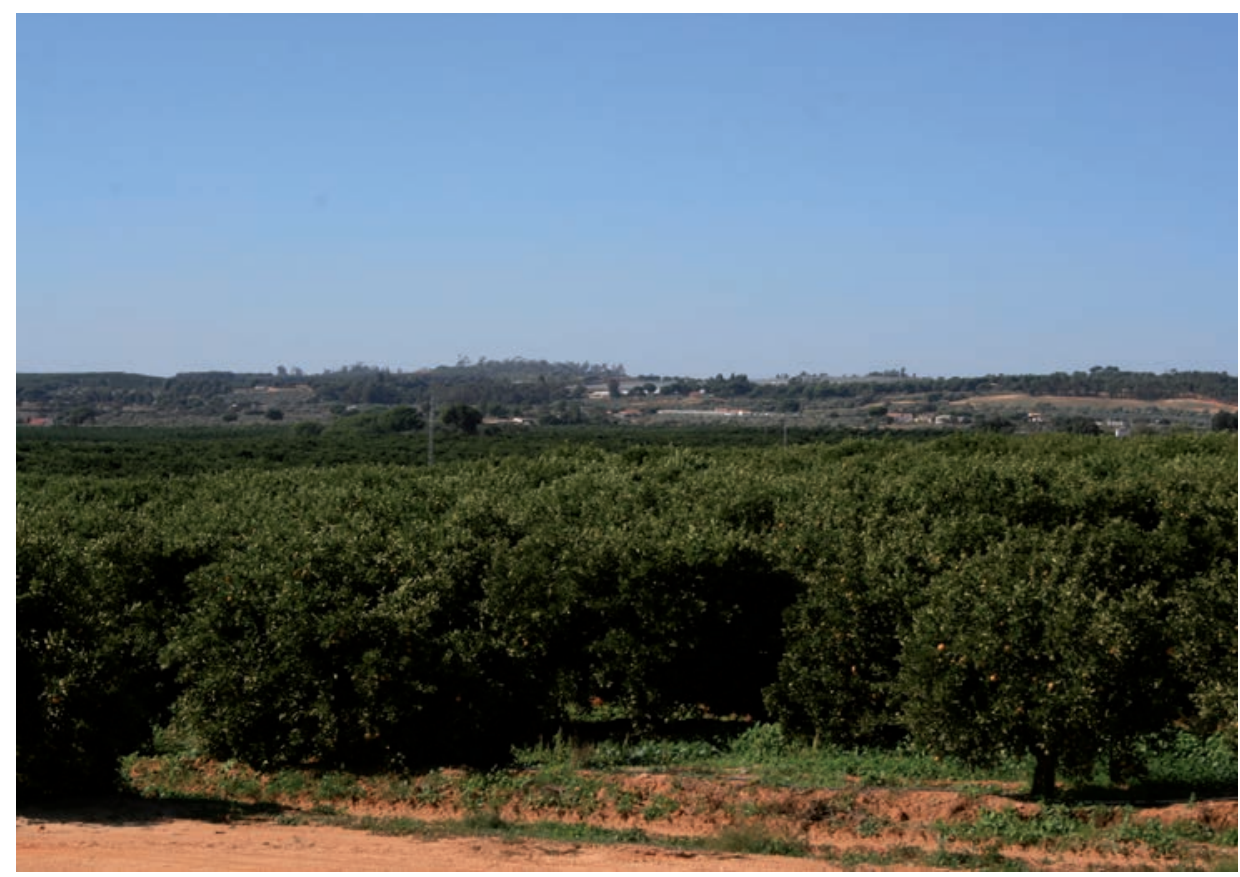

Foto 1. Unidad 1. Carretera San Bartolomé de la Torre - Gibraleón (Huelva). Descripción: Relieve llano en el primer plano, ocupado por un extenso cultivo de cítricos, y alomado en el segundo plano, ocupado por un entramado de olivar, vegetación natural y edificaciones. 


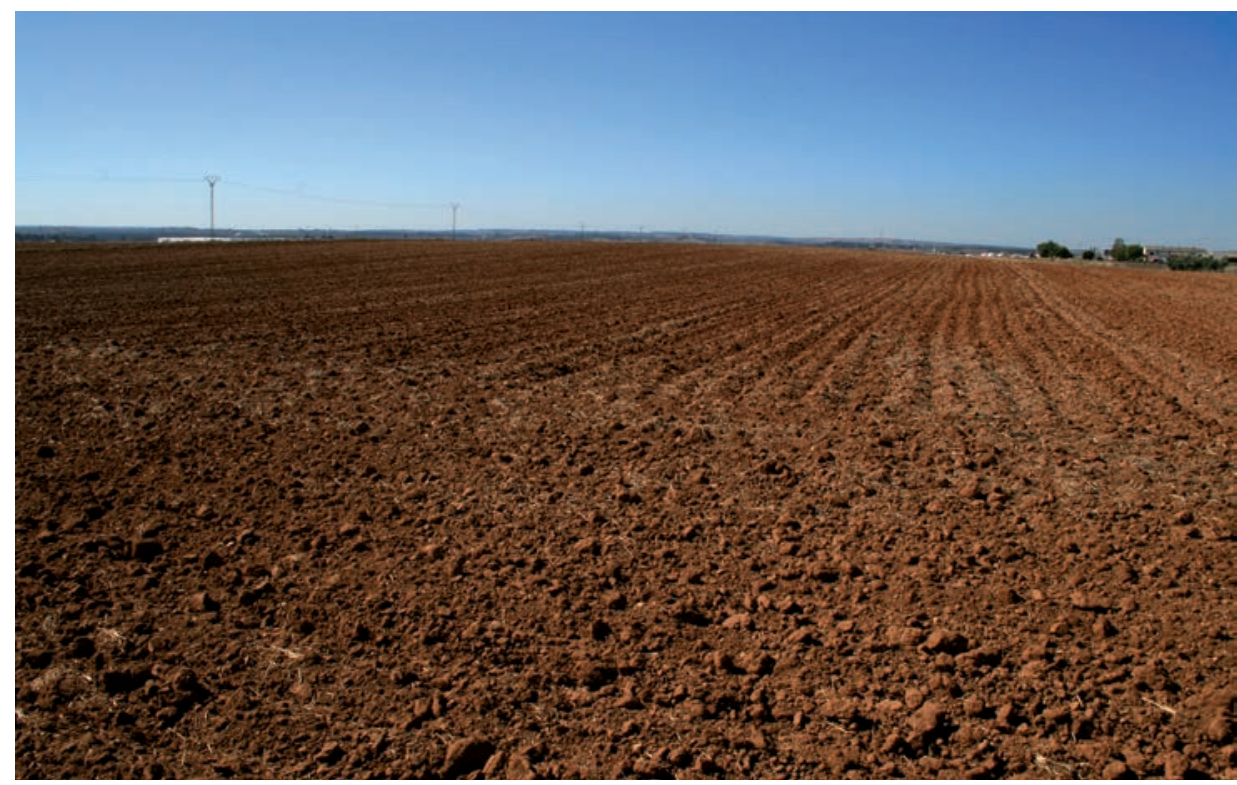

Foto 2. Unidad 2. Carretera Gibraleón-Trigueros (Huelva). Descripción: Relieve prácticamente llano sobre el que aparecen tierras roturadas dedicadas a cultivos herbáceos en secano dando como resultado un paisaje homogéneo.

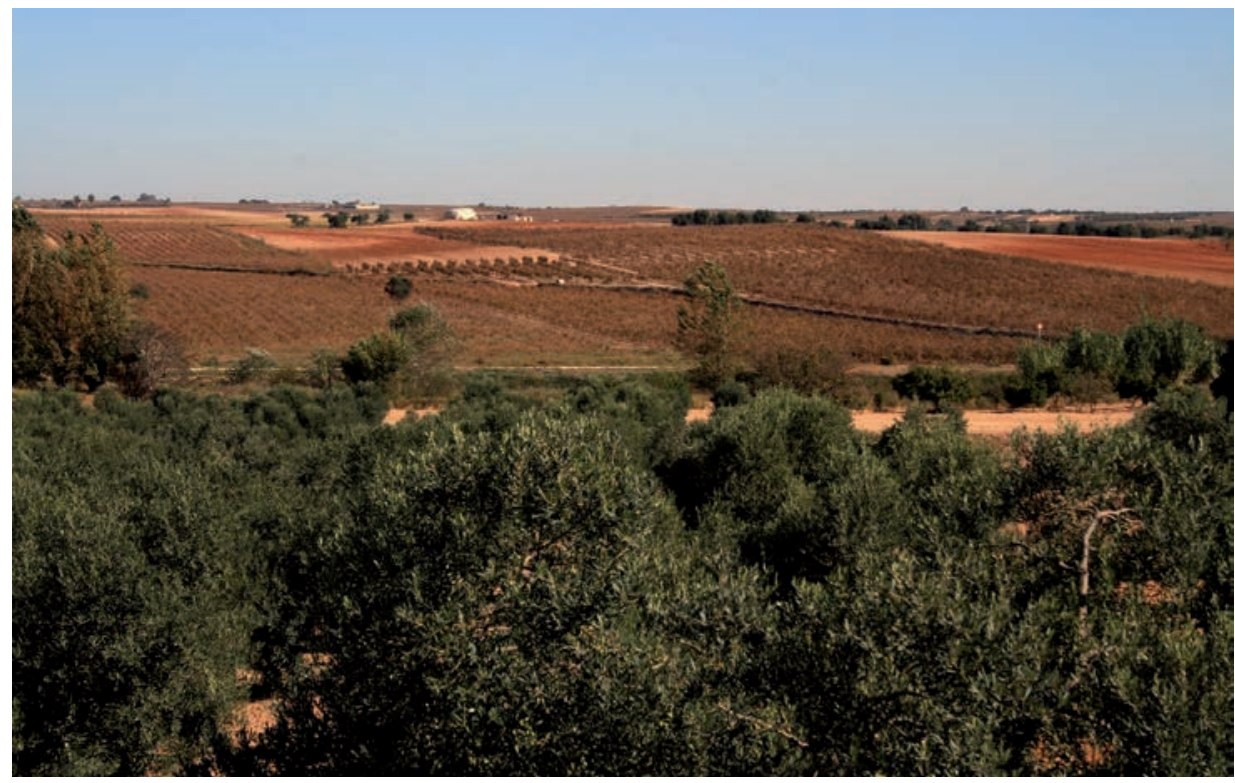

Foto 3. Unidad 3. Carretera de Almonte-Bonares (Huelva). Descripción: Paisaje muy fragmentado con un relieve suave, ligeramente alomado, donde aparecen viñedos, manchas de olivar, tierras dedicadas a cultivos herbáceos en secano y vegetación natural en los linderos. 
Según el Análisis Discriminante (Tabla 1) el grado de coincidencia global de la clasificación TWINSPAN y la realizada mediante las funciones discriminantes es del 82.24\%. La mayor coincidencia entre ambas clasificaciones se da para el G1 del TWINSPAN (grupo observado) (82.60\%) y la menor para el G3 (74.71\%). Las mayores diferencias en la asignación de cuadrículas a un grupo u otro entre la clasificación TWINSPAN y el Análisis Discriminante se dan entre los grupos 1 y 2 (31 y 57 cuadrículas afectadas) y los grupos 2 y 3 (71 y 55 cuadrículas afectadas). El hecho de que tan solo 5 cuadrículas identificadas como del G1 por el TWINSPAN aparecen en el Análisis Discriminante asociadas al G3 y 12 del G3 del TWINSPAN ubicadas en el G1 del Análisis Discriminante, evidencia que ambos grupos del TWINSPAN, G1 y G3, son los más nítidamente distinguibles entre sí, reforzándose así, indirectamente, la conclusión extraída a este respecto del análisis de la gráfica del DCA (Fig. 12). Sin embargo, en el caso del G3 los resultados del Análisis Discriminante no han sido coincidentes con los del TWINSPAN y el DCA, ya que este grupo ha sido el que mayor tasa de error ha presentado según el Análisis Discriminante, mientras que en el DCA es el grupo más compacto, y en el TWINSPAN, el que presenta las variables indicadoras con un valor indicador más elevado.

Tabla 1. Resultados del análisis discriminante.

\begin{tabular}{|c|c|c|c|c|}
\hline \multirow{2}{*}{ Grupo Observado } & \multicolumn{3}{|c|}{ Asignados a Grupo } & \multirow{2}{*}{$\begin{array}{c}\text { Total y } \\
\text { \% Correctos }\end{array}$} \\
\cline { 2 - 5 } & $\mathbf{1}$ & $\mathbf{2}$ & $\mathbf{3}$ & $207 / 82.60$ \\
\hline $\mathbf{1}$ & 171 & 31 & 5 & $869 / 80.66$ \\
\hline $\mathbf{2}$ & 57 & 701 & 71 & $265 / 74.71$ \\
\hline $\mathbf{3}$ & 12 & 55 & 198 & $1301 / 82.24$ \\
\hline
\end{tabular}

La fiabilidad de los resultados se evidencia por el error de estimación total obtenido a través de la validación cruzada: $19.37 \%$, frente al $17.76 \%$ que es el error de estimación del Análisis Discriminante.

\section{Discusión}

\subsection{Variables utilizadas}

La justificación de las variables utilizadas requiere de la toma en consideración de la extensión del ámbito de estudio y el marco en el que éste se ha realizado. El estudio se centra en la diferenciación de paisajes dentro de un tipo de paisaje identificado como Campiñas. Este tipo de paisaje (junto con otros 7 tipos distintos), fue reconocido como tal en una clasificación de paisajes de la provincia de Huelva (considerada en su totalidad), realizada previamente (Alcántara, 2008). En consecuencia, la extensión del territorio original de este estudio, $1301 \mathrm{~km}^{2}$, conlleva una escala de detalle que sitúa al 
mismo en el extremo opuesto a, por ejemplo, el mapa de los paisajes de Europa, realizado a escala 1:5000 000 (Mücher et al., 2003).

Aunque las variables que pueden utilizarse para clasificar paisajes son múltiples, es posible agruparlas en tres grandes grupos, abióticas, bióticas y culturales. Es, a su vez, posible, ordenar estas variables jerárquicamente, situando en los niveles más altos aquellas que son más independientes y que son, además, más determinantes respecto de otras, más dependientes, y situadas en niveles jerárquicos inferiores. Así, variables como el clima o la geología se sitúan en el nivel superior; la vegetación en niveles medios y los usos del suelo en niveles inferiores (Mücher et al., 2003). Niveles inferiores en los que deben considerarse también otros como patrones de asentamiento, patrones de distribución de la propiedad, etc. (Swanwick, 2002). A pesar de la dificultad de generalizar, es claro el predominio de los factores biofísicos en las clasificaciones de los paisajes que abarcan territorios de una cierta extensión, como se concluye en Wascher (2005), después del análisis de 49 clasificaciones europeas del paisaje de ámbito predominantemente nacional.

Dadas las características del territorio a estudiar, fundamentalmente su homogeneidad en relación a variables usadas de forma generalizada en las clasificaciones del paisaje a pequeña escala, se consideró que las variables seleccionadas podrían ser las más útiles en el proceso de diferenciación de paisajes. Así, por ejemplo, la utilización de la variable litología, empleada a nivel provincial, poco podía aportar a la sectorización paisajística dentro del tipo de paisaje objeto de estudio, Campiñas, al haber sido una variable de valor diagnóstico en la diferenciación de este tipo de paisaje frente a otros en la clasificación llevada a cabo a nivel jerárquico superior, por el predominio en Campiñas de las margas y su escasa representación en otros tipos de paisaje (Alcántara, 2008). Algo similar sucedía con las variables de altitud utilizadas, de valor diagnóstico a nivel provincial pero de utilidad dudosa en un territorio tan llano como el de Campiñas (particularidad ésta, la dificultad de la clasificación cuando las variaciones topográficas son menores, puesta ya de manifiesto por Bunce et al. (1996)). Por el contrario la variabilidad de los usos del suelo y coberturas vegetales de unas zonas a otras dentro de Campiñas, permitía suponer a priori su posible capacidad diagnóstica. Es, además, una variable que pone de manifiesto las actividades desarrolladas por el hombre sobre el territorio y que determinan, en parte, el paisaje. Es, por tanto, una variable cultural (con ese significado se ha utilizado por ejemplo en Chuman y Romportl (2010)), pero también una variable ambiental, no solo por la presencia de coberturas vegetales más o menos naturales, sino porque las características ambientales del territorio son determinantes de los usos del suelo posibles (incluidos los tipos de cultivos a desarrollar). Por último, es una de las características del territorio perceptibles visualmente y que, en una aproximación ecológica y sistémica al paisaje, integran el subsistema identificado como fenosistema (González Bernáldez, 1981). La variable municipios es una variable cultural en cuanto a artefacto, cuya impronta en el territorio es difícil de concretar, y, sobre todo, es una variable de referencia espacial, de vital importancia para la localización de los elementos a clasificar.

Por el tipo de relieve del territorio, la importancia de las variables de pendiente en los resultados ha sido menor. Sin embargo cabe destacar que en los niveles subsecuentes de división del TWINSPAN, el grupo de cultivos cerealistas predominantes, G2, se subdivide en dos atendiendo a variables de pendiente y de usos del suelo. De esta manera, 
aparecen dos subgrupos: uno de mayores pendientes, dominado por cultivos leñosos en secano: olivar (C15) y otro más llano dominado, por cultivos herbáceo en secano (C14). Estos resultados ponen de manifiesto lo que parece ser una forma lógica de aprovechamiento de las tierras de labrantío, en la que los secanos son aprovechados para el cultivo del cereal mientras la pendiente lo permite, siendo sustituidos, al incrementarse ésta, por el olivar. En cualquier caso, las diferencias entre estos subgrupos no se han considerado con entidad suficiente como para su reconocimiento e independización, habiéndose considerado sencillamente como una muestra del grado de heterogeneidad que pueden mostrar las unidades paisajísticas.

Por último, conviene resaltar que la caracterización íntegra de las unidades de paisaje reconocidas a escala de detalle, debe recoger no solo las variables indicadoras diagnósticas de las mismas (en este estudio las que han diferenciado las unidades 1, 2 y 3), sino también aquellas otras variables indicadoras que al nivel jerárquico superior han terminado conduciendo a la delimitación del paisaje analizado, en este caso Campiñas.

\subsection{La metodología: TWINSPAN}

El análisis de las ventajas/inconvenientes de la metodología seguida, el TWINSPAN, requiere de una primera consideración sobre el papel creciente de los métodos estadísticos de clasificación del paisaje, con el uso combinado de técnicas basadas en sistemas de información geográfica (SIG) y bases de datos digitales, lo que se concreta, por lo usual, en el desarrollo de un SIG con una configuración flexible (Mücher et al., 2003). Las ventajas fundamentales de estas metodologías de clasificación del paisaje, identificadas genéricamente como métodos objetivos (Bunce et al., 1996), pueden sintetizarse en la obtención de unos resultados más transparentes y comparables y, en definitiva, más objetivos y fiables (Wascher, 2005). A lo que cabe añadir las ventajas intrínsecas al uso de los SIG, como amoldar la información de salida a las necesidades, etc. (Mücher et al., 2003). En resumen, además de la posibilidad que ofrecen estas metodologías de realizar una clasificación jerárquica, las clasificaciones estadísticas ayudan a simplificar la heterogeneidad de los complejos patrones ambientales (Jongman et al., 2006).

El TWINSPAN es un método multivariante de clasificación que comparte en esencia las ventajas más arriba aludidas en relación al uso de los métodos estadísticos en asociación a SIG. Ha sido utilizado en otras clasificaciones del paisaje, además de su uso muy frecuente para la clasificación de muestras de vegetación, contexto en el que se desarrolló originalmente Haines-Young (1992), Bunce et al. (1996), McGarigal et al. (2000), Chuman y Romportl (2010) o VVAA (2014). Sin embargo presenta algunas características que no presentan otros métodos estadísticos de clasificación, entre las que deben destacarse tres de ellas.

Una primera es su capacidad para trabajar con bases de datos con elevado número de ceros, propias de estudios de vegetación y paisajísticos y que no todos los métodos multivariantes de clasificación poseen (Bunce et al., 1996). Una segunda es que conjuga la técnica de clasificación, cuyo resultado gráfico es el dendrograma (Fig. 10), con la de ordenación, cuyo resultado gráfico es la distribución de las muestras (las cuadrículas) en 
el espacio definido por las variables utilizadas (Fig. 12), así como la de las variables en el espacio muestral (Fig. 13). La recomendación como procedimiento de rutina en ecología del uso complementario de análisis de clasificación y diagramas de ordenación (Legendre y Legendre, 1998), se ve respaldada por los resultados que se han presentado. De los mismos se deriva que los gráficos de ordenación (Figs. 12 y 13) proporcionan una información sobre las tres unidades de paisaje diferenciadas que no se puede obtener a partir únicamente del dendrograma y que permite una visión más completa de las mismas.

Una tercera característica es la identificación de las variables más determinantes en la diferenciación de los grupos del TWINSPAN (Chuman y Romportl, 2010). Esta identificación va además asociada a un valor numérico, el valor indicador, que cuantifica el valor diagnóstico de la variable y, por tanto, el peso relativo de la misma en la identificación de la unidad de paisaje de que se trate. Si, como se reclama en los proyectos de clasificación del paisaje a nivel europeo realizados en tiempos recientes (Swanwick, 2002; Mücher et al., 2003; Wascher, 2005; Jongman et al., 2006), es primordial en un primer paso identificar y caracterizar los paisajes, y esta caracterización implica: "identifying, mapping, classifying and describing landscape character" (Swanwick, 2002), no puede sino concluirse la evidente utilidad de las variables indicadoras y sus valores asociados en este proceso de caracterización. Si bien se considera que un observador cualificado tras un recorrido detallado por el territorio de estudio, podría reconocer la distribución dispar de los cultivos herbáceos en secano, viñedos, etc., y su impacto en los paisajes resultantes, parece que sería más difícil que pudiera cuantificar cada una de estas variables y así ordenar las mismas según su mayor o menor peso para la diferenciación de los paisajes. Esta es, pues, una aportación fundamental de esta metodología, cuyo potencial explicativo se ha intentado poner de manifiesto en el apartado de Resultados.

La principal limitación del TWINSPAN deriva de su incapacidad para el manejo de más de 25000 objetos (Chuman y Romportl, 2010), limitación subsanada en ocasiones recurriendo a muestreos estratificados a fin de obtener una muestra $<25000$ cuadrículas (Bunce et al., 1996). De ahí que no sea una metodología adecuada para clasificaciones de los paisajes a pequeña escala, como la de Europa o la clasificación de los paisajes de España.

Ésta es una de las razones que explican, en parte, la diversidad de metodologías de clasificación del paisaje dentro del grupo de métodos estadísticos asociados a SIG. Así, el mapa de los paisajes de Europa, se basa en interpretaciones automatizadas de imágenes utilizando el software eCognition, que es un software de clasificación de imágenes orientado al objeto para el análisis multi-escalar de datos geográficos de todo tipo (Mücher et al., 2003; Wascher, 2005). La clasificación medioambiental de Europa, realizada en paralelo a la clasificación de los paisajes (Mücher et al., 2003) -y cuya segmentación en 13 zonas ambientales ha sido utilizada en el desarrollo de esta última (Wascher, 2005) - utiliza a su vez una metodología distinta. Las aportaciones más relevantes son: la utilización del píxel como elemento a clasificar y, sobre todo, la utilización de un único software, ISODATA, para el manejo de la información.

El espectro metodológico se amplía con la variedad de enfoques: estudios que abordan la clasificación de abajo a arriba (bottom-up) en vez de arriba a abajo (top-down), como se ha hecho en este estudio (Robitaille y Saucier, 1996); de objetivos -tipificación de las unida- 
des de paisaje en función de determinados atributos y funciones específicas de los paisajes, amenazas y riesgos presentes, objetivos ecológicos que han de cumplirse en cada unidad, etc. (Bastian, 2000); de nivel jerárquico-el énfasis en la identificación de elementos paisajísticos y biotopos más que de paisajes mediante la interpretación de fotografías aéreas de infrarrojo falso color (CIR) a escala 1:30 000 (Cousins y Ihse, 1998); o del uso o no de las cuencas visuales en la génesis de los mapas del paisaje (Burrough y De Veer, 1989).

\subsection{Validación}

La validación de los resultados de cualquier proceso de clasificación es considerada por Haines-Young (1992) como el aspecto más problemático al no existir un conjunto consensuado de criterios para el contraste de la clasificación obtenida. Sí está claramente asentada la necesidad de chequear la eficiencia de una clasificación con parámetros independientes, de manera que se complemente con exámenes objetivos e interactivos (Bunce et al., 1996; Wascher, 2005). Los métodos utilizados son múltiples: la comparación de los mapas generados con otros realizados previamente y de diferente autoría (Haines-Young, 1992; Mücher et al., 2003); análisis del grado de correlación de la clasificación con variables de distribución territorial (como distribución de especies de Quercus o de la Vegetación Potencial Natural en la clasificación medioambiental de Europa (Mücher et al., 2003)); uso de métodos estadísticos, como el uso del chi-cuadrado para evaluar el grado de asociación entre clasificaciones (Bunce et al., 1996), etc. Se considera también fundamental el trabajo de campo que permite refinar el resultado (Wascher, 2005) o, como sucede en el Atlas de los Paisajes de España (Sanz Herráiz et al., 2003), constituye un pilar fundamental de la delimitación y caracterización de los paisajes. En el caso de los paisajes de Campiñas diferenciados en este estudio, la validación se ha realizado, con un resultado satisfactorio, mediante una metodología encuadrable dentro del uso de los métodos estadísticos.

\subsection{Otras clasificaciones en España}

El análisis comparado del presente estudio y las dos publicaciones sobre paisajes españoles de mayor relevancia para el mismo, el Atlas de los Paisajes de España (Sanz Herráiz et al., 2003) y el Mapa de Paisajes de Andalucía (Moreira et al., 2005), permite concluir que existen notables diferencias. En primer lugar la extensión de los territorios estudiados, notablemente mayor en estos dos estudios, con todo lo que ello implica, como distinta escala de estudio o distintos niveles jerárquicos de la tipología paisajística (tres en ambos mapas y uno en el presente estudio). En segundo lugar las diferencias desde el punto de vista metodológico. Estas diferencias pueden sintetizarse recurriendo a los cuatro tipos de métodos reconocidos por Wascher (2005) en función del grado en el que el método utilizado se basa en la interpretación humana o en una aproximación analítica automática:

1. Interpretación humana experta.

2. Interpretación experta con ayuda de algún análisis automático.

3. Análisis altamente automatizado.

4. Análisis automatizado, junto con algún refinado interpretativo. 
En Wascher (2005) se identifica la clasificación del Atlas de los Paisajes de España (Sanz Herráiz et al., 2003) como tipo 2. En esta misma categoría se considera debe situarse también el Mapa de Paisajes de Andalucía (Moreira et al., 2005). Mientras que el presente estudio se identifica como tipo 4, ya que la clasificación se ha obtenido mediante un método automatizado (TWINSPAN) y ha sido sometida a un refinado interpretativo (depurado).

Aunque el Atlas de los Paisajes de España presenta unidades paisajísticas para el tipo de paisaje identificado como Campiñas Andaluzas, no se ha procedido a una comparación detallada con las unidades paisajísticas del paisaje Campiñas de este estudio, por la falta parcial de coincidencia entre un territorio y otro. De hecho la unidad de paisaje 1, situada más al oeste (Fig. 14) no ha sido identificada como un tipo de paisaje de campiña en ninguna de las otras dos clasificaciones. Para la parte del tipo de paisaje Campiñas Andaluzas perteneciente a la provincia de Huelva, el Atlas identifica tres unidades paisajísticas, aunque una de ellas escasamente representada en Huelva. En el área donde coinciden las dos clasificaciones, grosso modo, se puede decir que la unidad 3 de este estudio se corresponde con una de las unidades descritas en el Atlas (Campiña de Condado -campiña de viñedo y olivar-) y la unidad 2, con la otra (Campiña de Gerena-Trigueros -campiña cerealista-).

\section{Conclusiones}

En el marco, progresivamente mayor, de la utilización de métodos de clasificación estadísticos asociados a SIG, se ha podido comprobar mediante este estudio que el TWINSPAN es una herramienta de particular utilidad en el proceso de clasificación de unidades del paisaje a escala de detalle. En primer lugar por las características propias de este tipo de análisis ya comentadas en la discusión. En segundo lugar por razones ligadas a la escala: en territorios extensos presenta ciertas limitaciones, existiendo métodos alternativos que soslayan las mismas. Además es evidente el predominio de los factores ambientales físicos (clima, geología, relieve, suelos) en la diferenciación entre paisajes y tipos de paisajes a niveles jerárquicos superiores. Mientras que al nivel inferior juegan un papel primordial variables más dependientes de las antes mencionadas, como los usos y coberturas del suelo que, por otra parte, se considera que constituyen ya una cierta representación de las condiciones culturales (Chuman y Romportl, 2010), no tomadas en consideración a escalas de trabajo pequeñas. Como consecuencia de las características asociadas a los territorios del nivel jerárquico inferior (mayor homogeneidad en los factores físicos, mayor importancia del patrón de usos del suelos...), la diferenciación de paisajes puede resultar más difícil y, por tanto, la existencia de un método multivariante como el TWINSPAN puede ser de gran ayuda.

A lo antedicho cabe añadir la necesidad de profundizar en las clasificaciones del paisaje a nivel de detalle con la incorporación de variables culturales o históricas. Este nivel es el más adecuado para la inclusión de variables de este tipo, no utilizadas a niveles superiores por ser, en general, los fenómenos culturales (arqueológicos, históricos, arquitectónicos...) demasiado complejos para permitir una clasificación global válida a nivel internacional (Mücher et al., 2003). Mientras que el nivel regional es el más idóneo para detectar patrones consistentes relativos a los asentamientos, manejos del suelo, tra- 
diciones, etc. (Wascher, 2005). Es, pues, una tarea por realizar, una vez disponible una tipología de los paisajes a nivel europeo.

\section{Referencias}

Agger, P., Brandt, J. 1984. Registration methods for studying the development of small-scale biotope structures in rural Denmark. En Proceedings of the First International Seminar on Methodology in Landscape Ecological Research and Planning (vol. 2), J. Brandt, J., P. Agger (Eds.), Roskilde, Denmark, pp. 61-72.

Alcántara, J. 2008. Aplicación de métodos multivariantes y SIG para una clasificación del paisaje (Huelva, España). Tesis Doctoral. Facultad de Ciencias. Universidad de Córdoba. Córdoba.

Bastian, O. 2000. Landscape classification in Saxony (Germany) - a tool for holistic regional planning. Landscape and Urban Planning 50, 145-155.

Bunce, R.G.H., Barr, C.J., Clarke, R.T., Howard, D.C., Lane, A.M.J. 1996. ITE Merlewood Land Classification of Great Britain. Journal of Biogeography 23, 625-634.

Burrough, P.A., De Veer, A.A. 1984. Automated production of landscape maps for physical planning in the Netherlands. Landscape Planning in the Netherlands 11, 205-226.

Chuman, T., Romportl, D. 2010. Multivariate classification analysis of cultural landscapes: An example from the Czech Republic. Landscape and Urban Planning 98, 200-209.

Council of Europe. 2000. European Landscape Convention. Congress of Local and Regional Authorities of Europe.

Cousins, S.A.O., Ihse, M. 1998. A methodological study for biotope and landscape mapping based on CIR aerial photographs. Landscape and Urban Planning 41, 183-192.

Farina, A. 2006. Principles and Methods in Landscape Ecology. Towards a Science of Landscape. Springer, Dordrecht, The Netherlands.

Forman, R.T.T., Godron, M. 1986. Landscape Ecology. John Wiley and Sons, New York.

González Bernáldez, F. 1981. Ecología y Paisaje. Blume, Barcelona.

Haase, G. 1989. Medium scale landscape classification in the German Democratic Republic. Landscape Ecology 3, 29-41.

Haynes-Young, R.H. 1992. The use of remotely-sensed satellite imagery for landscape classification in Wales (U.K). Landscape Ecology 7, 253-274.

Jongman, R.H.G., Bunce, R.H.G., Metzger, M.J., Howard, D.C., Mateus, V.L. 2006. Objectives and applications of a statistical environmental stratification of Europe. Landscape Ecology 21, 409-419.

Kent, M., Coker, M. 1992. Vegetation description and analysis. A practical approach. CRC Press Inc., Corporate Blvd., N.W., Boca Raton, Florida.

Legendre, P., Legendre, L. 1998. Numerical Ecology. Second English Edition. Elsevier Science B.V., Amsterdam.

McGarigal, K., Marks, B.J., 1994. FRAGSTATS: Spatial Pattern Analysis Program for Quantifying Landscape Structure (version 2.0). Forest Science Department, Oregon State University, Corvallis.

McGarigal, K., Cushman, S., Stafford, S. 2000. Multivariate Statistics for Wildlife Ecology Research. Springer-Verlag, New York.

Manly, B.F.J. 1997. Randomization, Bootstrap and Monte Carlo Methods in Biology. Second Edition. Chapman \& Hall/CRC, London.

Moreira, J.M., Rodríguez, M., Zoido, F., Moniz, C., Venegas, C. y Rodríguez, J. 2005. Mapa de Paisajes de Andalucía. En Atlas de Andalucía, Tomo II. Cartografía ecológica y territorial, 
Consejería de Obras Públicas y Transportes, Consejería de Medio Ambiente, Junta de Andalucía, Sevilla.

Mücher, C.A., Bunce, R.G.H., Jongman, R.H.C., Klijn, J.A., Koomen, A.J.M., Metzger, M., Wascher, D.M. 2003. Identification and characterization of environments and landscape in Europe. Wageningen, Alterra, Alterra-rapport 832.

Muñoz Serrano, A. 2003. Estadística Aplicada Uni y Multivariante. Consejería de Agricultura y Pesca. Junta de Andalucía, Sevilla.

Rivas Martínez, S. 1987. Memoria del mapa de series de vegetación de España. I.C.O.N.A. Serie Técnica. Madrid.

Robitaille, A., Saucier, J.P. 1996. Land District, ecophysiographic units and areas: the Landscape Mapping of the Ministère des Ressources Naturelles du Québec. Environmental Monitoring and Assessment 39, 127-148.

Sanz Herráiz, C., Mata Olmo, R., Gómez Mendoza, J., Allende Álvarez, F., López Estebánez, N., Molina Delgado, P., Galiana Martín, L. 2003. Atlas de los Paisajes de España. Centro de publicaciones. Secretaria General Técnica. Ministerio de Medio Ambiente, Madrid.

SAS Institute 1990. SAS Procedures Guide, Release 6.03 Edition. Cary, North Carolina.

Swanwick, C. 2002. Landscape character assessment - Guidance for England and Scotland. Countryside Agency and Scottish Natural Heritage. Countryside Agency Publications.

Troll, C. 1966. Okologische Landschaftsforschung und Vergleinchende Hochgebirgsforschung. Erdkundliches Wissen. Schriflenfolge fur Forschung und Praxis. FET II, Wiesbaden.

VV.AA. 2014. Bases para la realización del Sistema Compartido de Información sobre el paisaje de Andalucía. Consejería de Medio Ambiente y Ordenación del Territorio, Junta de Andalucía, Sevilla.

Wascher, D.M. (Ed.). 2005. European Landscape Character Areas - Typologies, Cartography and Indicators for the Assessment of Sustainable Landscapes. Final Project Report as deliverable from the EU's Accompanying Measure project European Landscape Character Assessment Initiative (ELCAI), funded under the 5th Framework Programme on Energy, Environment and Sustainable Development.

Zonneveld, I.S. 1979. Land Evaluation and Land(scape) Science. Enschede, The Netherlands.

Zonneveld, I.S. 1989. The land unit - a fundamental concept on landscape ecology, and its applications. Landscape Ecology 3, 67-86. 